\title{
Does the resolving of clinical problems and doubts about medicines using WhatsApp by medicine and podiatry undergraduate students improve their learning and assessment?
}

\author{
Inmaculada Bellido, Encarnación Blanco ${ }^{1}$, M Victoria Bellido², Jose Luis Guerrero-Orriach ${ }^{3}$, \\ Aurelio Gomez-Luque ${ }^{3}$
}

${ }^{I}$ Department of Pharmacology and Clinical Therapeutics, School of Medicine, IBIMA, University of Malaga, Spain, ${ }^{2}$ Emergency surgery service, Carlos Haya-Materno Infantil Regional Universitary Hospital, School of Medicine, IBIMA, University of Malaga, Spain, ${ }^{3}$ Anaesthesia service, Virgen de la Victoria Regional Universitary Hospital, School of Medicine, IBIMA, University of Malaga, Spain

Mobil phone use has become an indispensable part of young people's daily life. Mobile phones (in addition to conversations, photos and messages) can be a great tool of communication and dissemination. These small, handy technological devices have provided unique opportunities for use in teaching pharmacology including internet knowledge acquisition or downloaded applications, e-learning, or team member communication. As more and more students are using WhatsApp applications, it may have an important impact on the individual and collaborative learning. We aim to evaluate the impact of resolving clinical problems and doubts through WhatsApp in the learning of medicines used by undergraduate students on the Anaesthesia course and Pharmacology course of Medicine and Podiatry Degrees.

Methods. Prospective controlled study with students of Anaesthesia course from the Medicine Degree and Pharmacology course from the Podiatry Degree which were invited to voluntarily participate into a WhatsApp group to asking clinical questions and doubts about analgesic/anaesthetic drugs and to answer them. Clinical problems and doubts were formulated by the students and they were most commonly delivered by other students or. in non-answer case by teachers. All WhatsApp's comments were controlled by teachers. WhatsApp user group was compared with a non-WhatsApp user group.

Results and Discussion. Students of Anaesthesia course from the Medicine Degree and Pharmacology course from the Podiatry Degree, N 235, 75.7\% female, 20+/-3.1 years old, were enrolled. The percentage of questions answered and failed of the test final assessment after having been reviewed in WhatsApp and those obtained in WhatsApp not-reviewed questions were compared. Contributions' mean to issues raised-resolved through WhatsApp was 31.2+/-17 (Min 3 times/Max 73 times). The correct answered questions (in percentage) at the student's final assessment test were $71.4 \%$ in the question reviewed by the WhatsApp group vs. $47.4 \%$ in the non-reviewed by the WhatsApp-group (control) $(\mathrm{p}<0.5)$. If you cannot against technology, at least use it to your advantage and to your students' advantage.

Conclusión. Resolving clinical problems and doubts through WhatsApp improved the assessment final score of questions about medicines used in undergraduate students on the Anaesthesia course of Medicine Degree and on the Pharmacology course of Podiatry Degrees. 